\title{
Learned EEG-based brain self-regulation of motor-related oscillations during application of transcranial electric brain stimulation: feasibility and limitations
}

\section{Surjo R. Soekadar ${ }^{1,2,3}$, Matthias Witkowski ${ }^{1,2,3}$, Eliana G. Cossio ${ }^{2,3}$, Niels Birbaumer ${ }^{3,4}$ and Leonardo G. Cohen ${ }^{1}$}

${ }^{1}$ Human Cortical Physiology and Neurorehabilitation Section, NINDS, NIH, Bethesda, MD, USA

${ }^{2}$ Applied Neurotechnology Lab, Department of Psychiatry and Psychotherapy, University Hospital Tübingen, Tübingen, Germany

${ }^{3}$ Institute of Medical Psychology and Behavioral Neurobiology, University of Tübingen, Tübingen, Germany

${ }^{4}$ Ospedale san Camillo, IRCCS, Venice, Italy

\section{Edited by:}

Andreas Meyer-Lindenberg, Central Institute of Mental Health, Germany

\section{Reviewed by:}

Alexander Fingelkurts, BM-Science

- Brain and Mind Technologies

Research Centre, Finland

Andrew A. Fingelkurts, BM-Science

- Brain and Mind Technologies

Research Centre, Finland

\section{${ }^{*}$ Correspondence:}

Surjo R. Soekadar, Applied

of Psychiatry and Psychotherapy,

University Hospital of Tübingen,

Calwerstr. 14, 72076 Tübingen,

Germany

e-mail:surjo.soekadar@

uni-tuebingen.de
Neurotechnology Lab, Department

Objective: Transcranial direct current stimulation (tDCS) improves motor learning and can affect emotional processing and attention. However, it is unclear whether learned electroencephalography (EEG)-based brain-machine interface (BMI) control during tDCS is feasible, how application of transcranial electric currents during BMI control would interfere with feature-extraction of physiological brain signals and how it affects brain control performance. Here we tested this combination and evaluated stimulation-dependent artifacts across different EEG frequencies and stability of motor imagery-based BMI control.

Approach: Ten healthy volunteers were invited to two BMI-sessions, each comprising two 60-trial blocks. During the trials, learned desynchronization of mu-rhythms $(8-15 \mathrm{~Hz})$ associated with motor imagery (MI) recorded over $\mathrm{C} 4$ was translated into online cursor movements on a computer screen. During block 2, either sham (session A) or anodal tDCS (session B) was applied at $1 \mathrm{~mA}$ with the stimulation electrode placed $1 \mathrm{~cm}$ anterior of $\mathrm{C} 4$.

Main results: tDCS was associated with a significant signal power increase in the lower frequencies most evident in the signal spectrum of the EEG channel closest to the stimulation electrode. Stimulation-dependent signal power increase exhibited a decay of $12 \mathrm{~dB}$ per decade, leaving frequencies above $9 \mathrm{~Hz}$ unaffected. Analysis of BMI control performance did not indicate a difference between blocks and tDCS conditions.

Conclusion: Application of tDCS during learned EEG-based self-regulation of brain oscillations above $9 \mathrm{~Hz}$ is feasible and safe, and might improve applicability of $\mathrm{BMI}$ systems.

Keywords: brain-machine interface (BMI) control, motor imagery, EEG, transcranial electric stimulation (TES), stimulation artifacts

\section{INTRODUCTION}

Brain-machine interfaces (BMI) translate physiological features of brain activity associated with the user's intention or state into control signals of a particular device or computer (Birbaumer and Cohen, 2007). BMIs are increasingly used in the context of neurorehabilitation, e.g., after stroke (Ang et al., 2011; RamosMurguialday et al., 2013) with two main purposes: (1) to drive assistive devices or computers that surrogate a lost or impeded function, or (2) to facilitate motor recovery related to BMIbased neurofeedback training (Wang et al., 2010; Soekadar et al., 2011). The best-established BMI in stroke is based on volitional modulation of mu-rhythm $(8-15 \mathrm{~Hz}$, also called sensorimotor rhythm, SMR) recorded over the sensori-motor cortex using electro- or magnetoencephalography (EEG/MEG) (Buch et al., 2008; Ramos-Murguialday et al., 2013). Motor planning, imagery or execution is associated with a decrease in amplitude of mu-rhythm (Pfurtscheller and Neuper, 1997; Llanos et al., 2013). This decrease can be quantified as event-related desynchronization (ERD) (Pfurtscheller and Aranibar, 1979) and used by a BMI system to control an exoskeleton moving a patient's paralyzed hand (Soekadar et al., 2011).

Depending on the physiological features, several sessions of BMI training are typically required to achieve stable and reliable BMI control (Soekadar et al., 2011). Training-related BMI learning, however, is often substantially impeded in patients with brain lesions, such as stroke, traumatic brain injury or other brain disorders resulting in compromised learning capacity (Buch et al., 2008). Thus, it would be desirable to identify strategies to improve BMI learning and performance in these populations.

Several studies suggest that the application of weak electric currents in the form of transcranial direct current stimulation (tDCS) can improve motor learning (Zimerman et al., 2012), 
cognition (Metuki et al., 2012), memory consolidation (Dockery et al., 2009; Reis et al., 2009) and emotional processing (Nitsche et al., 2012). While the underlying physiological mechanisms of these effects are still unknown, it has been shown that tDCS can result in polarity-dependent shifts of membrane potentials and modulate cortical excitability (Nitsche and Paulus, 2000; Pellicciari et al., 2013). Recent work indicates that anodal tDCS applied before a motor imagery-based BMI session might improve BMI control (Wei et al., 2013) and modulate motor imagery-related mu-ERD (Matsumoto et al., 2010). A series of experiments suggests that learning is faster when tDCS is applied during a task compared to when it is applied prior to the task (Stagg et al., 2011). It was concluded that such timingdependency might be of particular importance for the development of plasticity-inducing stimulation protocols (Nitsche et al., 2007). Furthermore, in addition to being time-saving in the clinical context when applied during motor or BMI training, direct effects of tDCS on brain oscillatory activity in the context of such training can be assessed, allowing for a better understanding of how transcranial electric currents modulate task-related brain activity.

While a novel strategy using magnetoencephalography (MEG) for in vivo assessment of neuromagnetic brain oscillations during transcranial electric brain stimulation was recently introduced (Soekadar et al., 2013a), the immobility and costs of MEG constrains broader clinical applications, e.g., for BMI training in the context of neurorehabilitation. EEG, in contrast, is inexpensive and widely used in clinical environments. However, the application of electric currents at voltages of up to 20 volts to the human head while recording EEG in the range of millivolts is particularly challenging. This is due to two main reasons: (1) Most EEG systems use wet electrodes to improve conduction between electrodes and the skin. Likewise, the application of electric currents to the head also requires good conduction, mostly provided by using electrolyte gels or pastes. If the conductive agents used for the EEG electrodes and stimulating electrodes are in direct contact, the stimulation currents will saturate the EEG amplifier and impede any physiological recordings. (2) Electric stimulation of the head can be associated with additional stimulation-depended signals picked up by the EEG amplifiers that might reduce classification accuracy in BMI control. This would be disadvantageous for both assistive and neurofeedback BMI applications. While denoting any stimulation-dependent signal during neurophysiological recordings of brain activity as artifacts or noise (i.e., an irregular fluctuation of the measured signal that does not contain meaningful information or obscures the information of interest) is somewhat appealing, it should be emphasized that the separation between the physiological responses or effects of the stimulation and stimulator-dependent noise is difficult. While recently systems were introduced that integrate electric stimulation and recording of EEG (e.g., Starstim ${ }^{\circledR}$ by Neuroelectrics, Barcelona, Spain) allowing for simultaneous EEG monitoring during tDCS (Schestatsky et al., 2013), it remained unclear how EEG signals across different frequency bands recorded at different distances from the stimulating electrode are affected by tDCS, and whether learned electroencephalography (EEG)-based brain-machine interface (BMI) control e.g., using motor imagery (MI) during tDCS is feasible, reliable, and safe.
Here, we investigated such a combination choosing an electrode montage that places the stimulation electrode as close as $1 \mathrm{~cm}$ to the EEG electrode recording brain oscillations used for BMI control (see Figure 1A). To allow generalization to other BMI paradigms, we first characterized stimulation-dependent signals at different EEG locations. We then compared the signal power in different frequencies (delta: $0.1-4 \mathrm{~Hz}$; theta: $4-9 \mathrm{~Hz}$; alpha: 9-15 Hz; and beta: $15-30 \mathrm{~Hz}$ ) before stimulation and during stimulation, and identified those frequencies significantly influenced by stimulation-dependent signals. Finally, we calculated the effect size of the stimulation conditions for each frequency band. Potential side effects of stimulation, like tingling, itching or burning (Brunoni et al., 2011) were assessed throughout the sessions.

\section{MATERIALS AND METHODS PARTICIPANTS AND EXPERIMENTAL DESIGN}

Ten healthy volunteers (5 males, 5 females, mean age: 26.7 years \pm 4.3 ) exhibiting reliable and stable motor imagery-based BMI control in a previous investigation (Soekadar et al., 2011) were invited for two BMI sessions on consecutive days. All participants were right handed according to the Edinburgh Handedness Inventory (Oldfield, 1971). Each session was divided into two blocks of 60 trials (block 1, block 2). While no stimulation was delivered during block 1 of both sessions, either sham stimulation (session A) or anodal stimulation (session B) was applied during block 2. Sessions were conducted in random order. All participants gave written informed consent before entering the study. The study protocol was approved by the National Institute of Neurologic Disorders and Stroke Institutional Review Board (NINDS IRB).

\section{ELECTROENCEPHALOGRAPHIC (EEG)-RECORDINGS AND FEEDBACK OF mu-EVENT-RELATED DESYNCHRONIZATION (mu-ERD)}

Participants were seated comfortably in an armchair facing a computer monitor. EEG was recorded from the following conventional EEG-recording sites (F3, FC5, C3, P3, Fz, AFz, Cz, FCz, $\mathrm{F} 4, \mathrm{FC} 6, \mathrm{C} 4, \mathrm{P} 4$ according to the international 10/20 system) using a 12-channel active electrode EEG system (Acti-cap ${ }^{\circledR}$ and BrainAmp MRplus ${ }^{\circledR}$, BrainProducts, Gilching, Germany) with the reference electrode placed at $\mathrm{FCz}$ and the ground electrode at AFz. For translation of neurophysiological signals into visual feedback, BCI2000, a multipurpose standard BCI platform, was used (Schalk et al., 2004).

All participants were instructed to use visuo-kinesthetic MI of moving their left hand modulating right-hemispheric murhythms when they saw the visual cue indicating the initialization of each trial.

To rule out overt movements during MI, electromyography (EMG) was recorded from the first dorsal interosseus muscle (FDI), extensor digitorum communis (EDC), extensor carpi ulnaris (ECU) and flexor carpi radialis (FCR) during the sessions. Skin/electrode resistance was kept below $10 \mathrm{k} \Omega$. EMG signals were recorded at a sampling rate of $1 \mathrm{kHz}$ and high-pass filtered at $2 \mathrm{~Hz}$ (BrainAmp ExG ${ }^{\circledR}$, Brainproducts, Gilching, Germany). Trials in which EMG activity exceeded that recorded during rest by two standard deviations were interrupted and excluded from further analysis. The number of excluded trials in which 

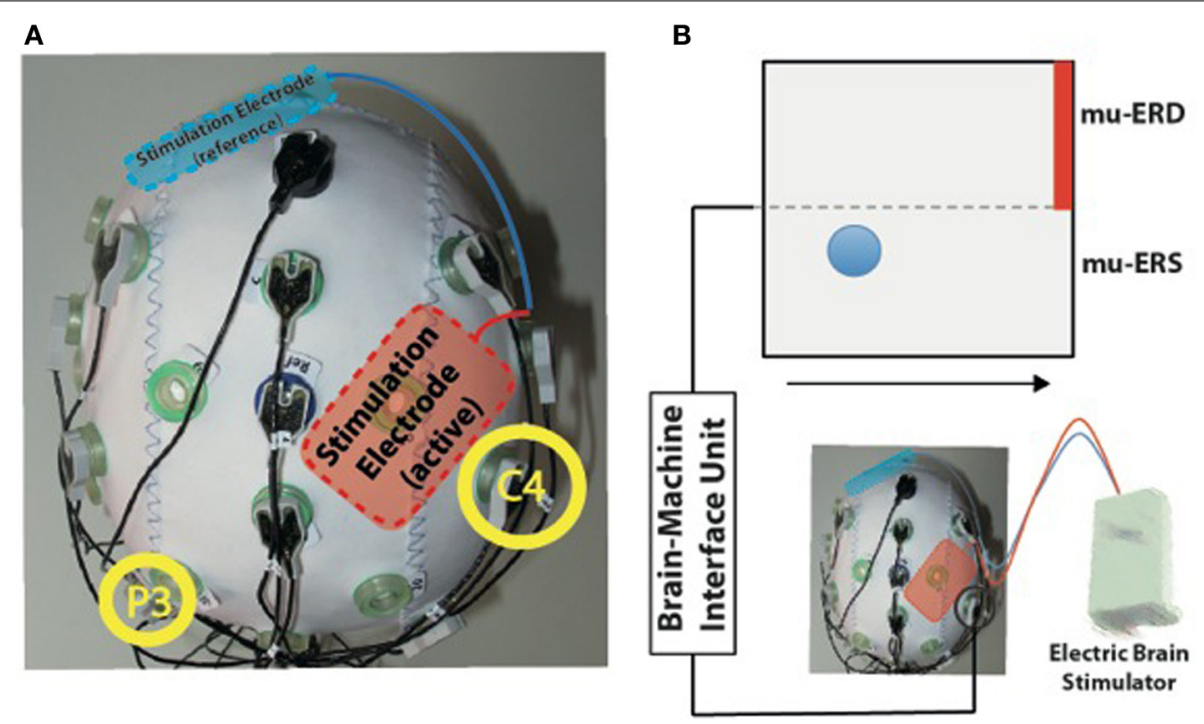

FIGURE 1 | (A) Experimental setup for simultaneous transcranial electric current stimulation during electroencephalography (EEG). The active stimulation electrode was placed immediately anterior to the EEG electrode C4 used for online brain-machine interface (BMI) control (right yellow circle). The reference stimulation electrode was placed over the left supraorbital region (blue). (B) BMI paradigm. Electric brain activity recorded at electrode C4 was translated into visual feedback. Event-related desynchronization of mu-rhythms $(9-15 \mathrm{~Hz}$, mu-ERD) was indicated by upward movements of a blue ball, while mu-event-related synchronization was indicated by downward movements (mu-ERS). Participants were instructed to keep the ball above the dotted horizontal line during the task to hit the target (indicated by red bar).
EMG activity was present was comparable across participants and ranged between 5 and $10 \%$.

Amplitude of mu-rhythm event-related desynchronization (mu-ERD) and synchronization (mu-ERS) was visually fed back to the participants during each trial (see Figure 1B). While increasing mu-ERD was indicated by up-movements of a ball above the horizontal midline of the screen, mu-ERS resulted in down-movements below the midline. Visual feedback was continuously updated every $100 \mathrm{~ms}$. Computation of mu-ERD/muERS included the power spectrum estimation (an autoregressive model of order 16 using the Yule-Walker algorithm) of each incoming sample at the optimal frequency for mu-ERD detection. The optimal frequency was identified in a screening run before the first session $(11 \mathrm{~Hz}$ in all participants). Resulting values were compared with mean power values of the preceding inter-trial-intervals (ITI) that were continuously updated during BMI control according to the method of Pfurtscheller and Aranibar (1979) and as previously implemented into a mu-ERD BMI system (Soekadar et al., 2011):

$$
\begin{aligned}
\bar{R} & =\frac{1}{N} \sum_{t=1}^{N} R_{t} \\
\operatorname{ERD}(t) & =\frac{T_{t}}{\bar{R}}-1
\end{aligned}
$$

Where $t$ represents the recorded sample block, $T_{t}$ the eventrelated task condition period and $R_{t}$ the power estimate in a given frequency band of $t . \bar{R}$ (reference value) represents power estimates during the rest (task-free) condition.
Each session consisted of 2 blocks with 60 trials. After the first block either sham stimulation (session A) or anodal stimulation (session B) was applied during the following 60 trials in a randomized order.

\section{TRANSCRANIAL DIRECT CURRENT STIMULATION (tDCS)}

tDCS was applied via two conducting $4 \times 6 \mathrm{~cm}$ rubber electrodes and attached to the participant's head using a conductive paste $\left(\right.$ Ten $20^{\circledR}$, D.O. Deaver, Aurora, CO, USA). The adhesive features of this paste prevented any sliding or dislocation of the electrodes during the attachment of the EEG cap. A bipolar electrode montage (right $\mathrm{M} 1$ and left supraorbital area) was used (see Figure 1A) to deliver a current of $1 \mathrm{~mA}$ (current density $0.04 \mathrm{~mA} / \mathrm{cm}^{2}$; total charge $0.048 \mathrm{C} / \mathrm{cm}^{2}$ using the DCSTIMULATOR PLUS, neuroConn GmbH, Germany). During sham stimulation, the DC stimulator was set up to apply an anodal current for $15 \mathrm{~s}$ and-at the offset-decrease stimulation intensity in a ramp-like fashion, a method shown to achieve a good level of blinding (Gandiga et al., 2006). Prior to the first block of the first session, the M1 hand-area was localized in all participants based on the motor evoked potential (MEP) hotspot of the first digit's interosseus muscle (FDI) using transcranial magnetic stimulation (TMS). After the end of block 2 of sessions $\mathrm{A}$ and $\mathrm{B}$, all participants rated possible discomfort, pain, tingling, itching or burning associated with the stimulation on visual analog scales (VAS) to assess safety and tolerability of tDCS during BMI control.

\section{OFFLINE ANALYSIS}

For all outcome measures, assumption of a normal distribution (Shapiro-Wilk test of normality) was tested. Parametric 
tests were corrected by Greenhouse-Geisser estimates if Mauchly's sphericity test indicated significance. To compare signal power across conditions, fast Fourier transformations were performed for all EEG data collected during the first and second blocks of session A and session B (see Figure 2). A repeated-measures ANOVA (rmANOVA) with factors "block" (block 1, block 2) and "frequency" (delta, theta, alpha, beta) was performed based on the raw EEG signal power recorded from electrode C4 (in immediate proximity to the stimulation electrode) and P3 (at $\sim 8-10 \mathrm{~cm}$ distance from the stimulation electrode) to investigate stimulation-dependent changes in different EEG channels. Posthoc paired-samples Students $t$-tests were used when applicable and corrected for multiple comparisons (Bonferroni). Timefrequency representations (TFR) were plotted for both conditions and tested for statistical differences at two different electrode positions ( $\mathrm{C} 4$ in immediate proximity of the stimulation electrode, and P3) using a cluster-based permutation test (Maris and Oostenveld, 2007). Effect size of stimulation-dependent signal differences in each frequency band was calculated using Cohen's $d$ transformed into a regression coefficient $r$ where $r<0.3$ is considered a small, $r<0.5$ is considered a medium, and $r>0.5$ is considered a large effect (Cohen, 1988). A rmANOVA with factors "session" (session A, session B) and "block" (block 1, block 2) was used to evaluate changes of BMI control across sessions in the absence (block 1) and presence (block 2) of anodal tDCS. BMI control was defined as the time during each trial in which the ball was above midline (indicating mu-ERD). All analyses were performed in SPSS 17.0. Significance level was set to $p<0.05$. Variance is defined as the standard error of the mean.

\section{RESULTS}

STIMULATION-DEPENDENT CHANGES OF SIGNAL POWER ACROSS DIFFERENT FREOUENCY BANDS AND CONDITIONS

Stimulation electrode in close proximity $(\sim 1 \mathrm{~cm})$ to the recording EEG channel (C4)

While rmANOVA of data recorded during session A (sham stimulation during block 2) from electrode C4 (Figure 2A) showed a main effect for "frequency" $\left[F_{(1,27)}=37.156, p<0.0001\right]$, indicating an expected difference between power values across the investigated frequency bands, there was no effect for "block" $\left[F_{(1,27)}=0.233, p=0.641\right]$ and no interaction between the factors $(p=0.188)$. Post-hoc $t$-tests showed no significant differences between power values of block 1 and 2 in any frequency band (delta: $p=0.729$; theta: $p=0.963$; alpha: $p=0.946$; beta: $p=0.784)$.
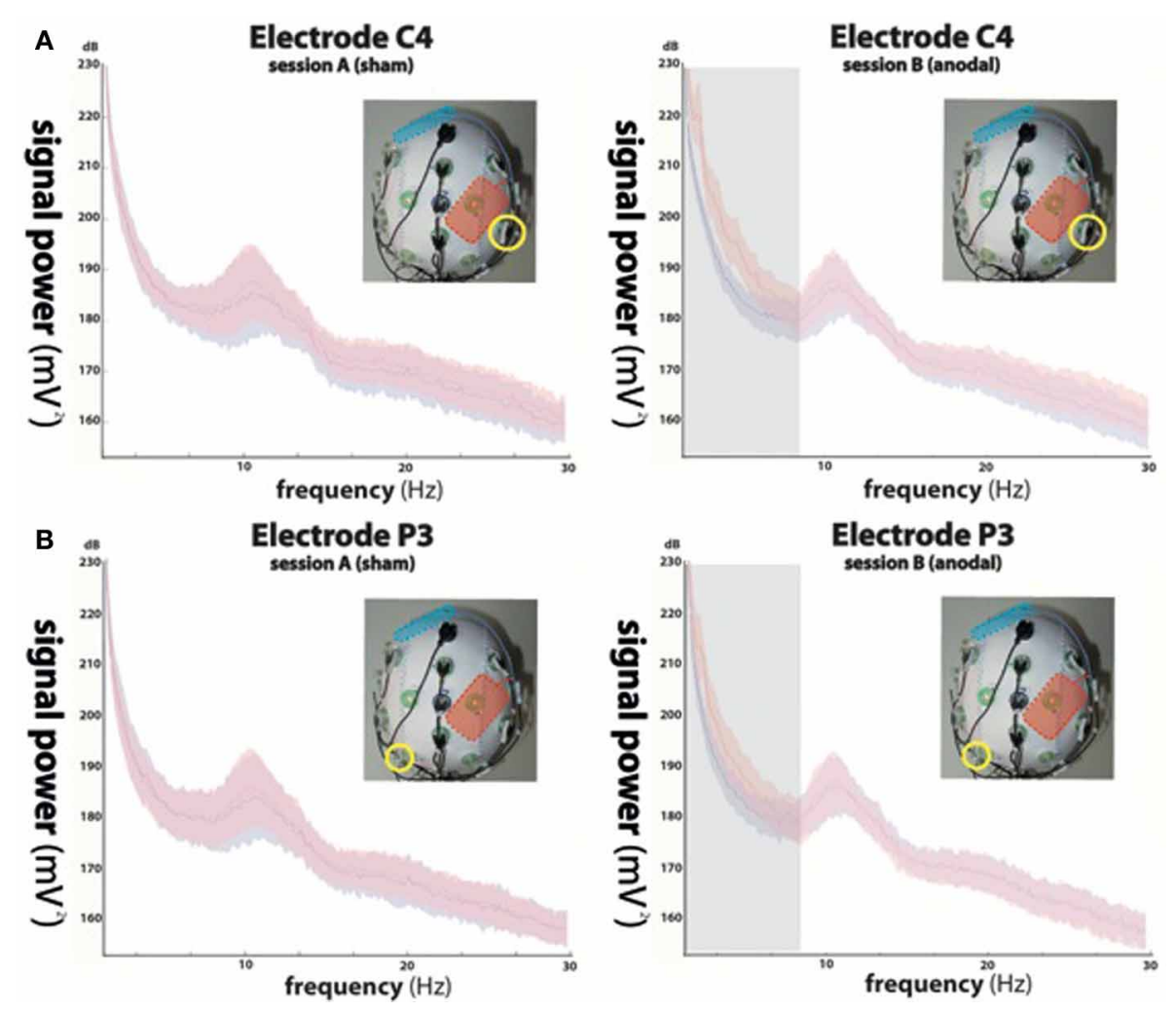

FIGURE 2 | Power spectra of the electroencephalographic (EEG) signals recorded from electrode $C 4(A)$ indicated by yellow circles in the upper panels and P3 (B) during sham stimulation (left column, red curve) and anodal stimulation (right column, red curve). Power spectra of trials in absence of stimulation (block 1) are shown in blue. While not significant during sham stimulation (left column), anodal stimulation 
When analyzing data of session B (anodal stimulation during block 2) from the same location, we found a main effect for both "frequency" $\left[F_{(1,27)}=77.536, p<0.0001\right]$ and "block" $\left[F_{(1,27)}=33.16, p<0.0001\right]$ and an interaction between the two $\left[F_{(1,3)}=39.584, p<0.0001\right]$. Post-hoc $t$-tests showed a significant difference between power values of block 1 and 2 in the delta band $(p<0.0001)$ and a trend in the theta band $(p=0.068)$, but no significant differences in the alpha $(p=0.482)$ or beta (beta: $p=0.336)$ bands.

\section{Stimulation electrode at $\sim 8-10 \mathrm{~cm}$ distance to the recording EEG channel (P3)}

Analysis of EEG data recorded $\sim 8-10 \mathrm{~cm}$ away from the stimulation electrode (P3) (Figure 2B) during session A, revealed a main effect for "frequency" $\left[F_{(1,27)}=28.28, p<0.0001\right]$, but no effect for "block" $\left[F_{(1,27)}=1.202, p=0.301\right]$ and no interaction between the factors $(p=0.056)$. We found no significant differences between power values of block 1 and 2 in any frequency band (delta: $p=0.655$; theta: $p=0.984$; alpha: $p=0.937$; beta: $p=0.923)$.

The same analysis performed for data acquired during session B (anodal stimulation during block 2) at electrode position P3, showed a main effect for both, "frequency" $\left[F_{(1,27)}=34.39, p<\right.$ $0.0001]$ and "block" $\left[F_{(1,27)}=11.34, p<0.01\right]$ and a significant interaction between the two $\left[F_{(1,3)}=14.152, p<0.0001\right]$. Posthoc $t$-tests indicated a significant difference between power values of block 1 and 2 in the delta band $(p<0.05)$, but not in the theta $(p=0.217)$, alpha $(p=0.445)$ or beta (beta: $p=0.482)$ bands.
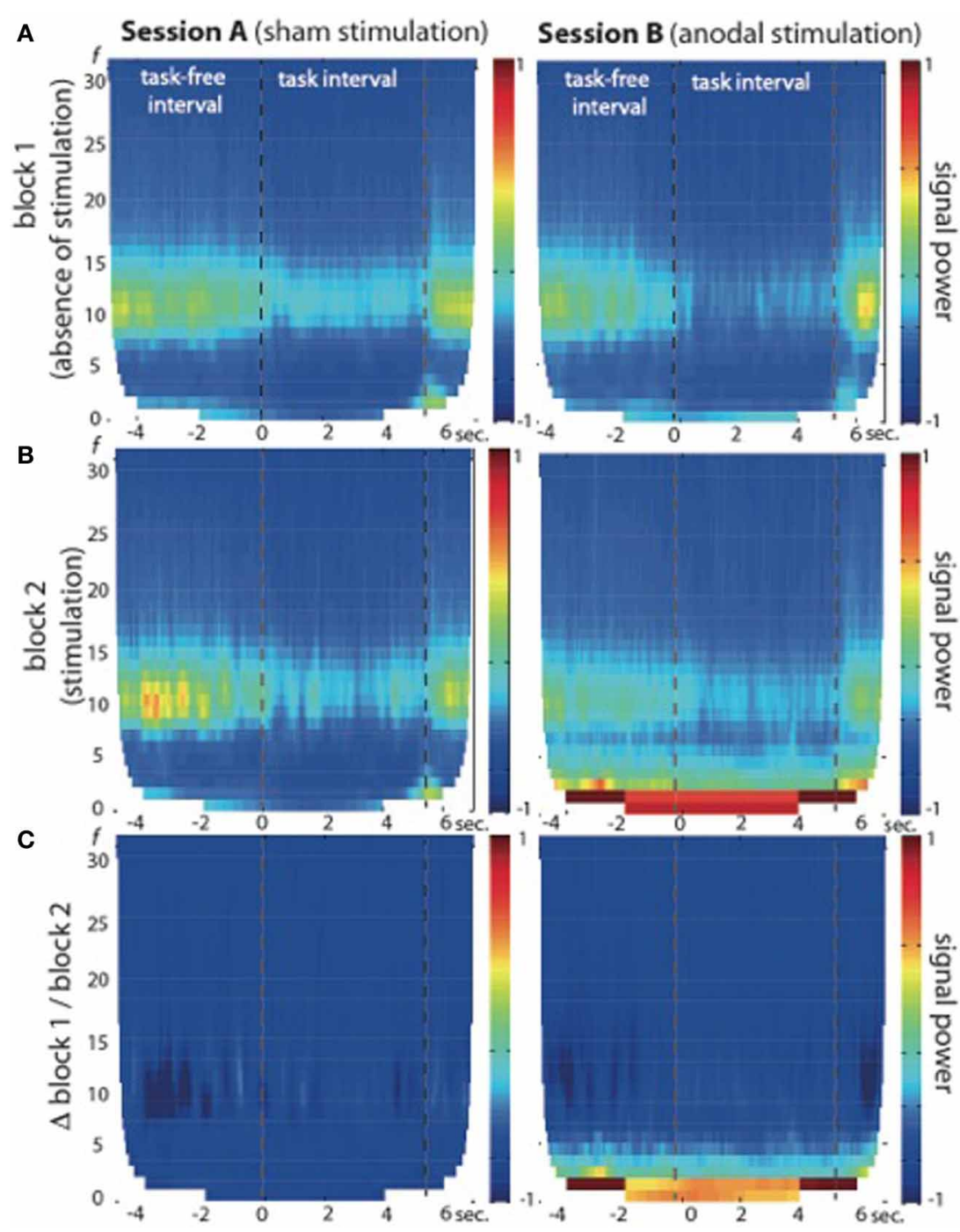

FIGURE 3 | Time-frequency representation (TFR) of brain oscillations recorded from EEG electrode position $\mathrm{C4}$ at $1 \mathrm{~cm}$ distance from the active electric brain stimulation electrode. (A) Block 1 of session A (left) and session B (right) in absence of electric brain stimulation. (B) Block 2 of session A (left, during sham stimulation) and session B (right, during anodal stimulation). Note the signal power increase in frequencies below $9 \mathrm{~Hz}$ in block 2 of session $\mathrm{B}$ (during anodal stimulation) across task-free and task intervals.

(C) Signal power differences between block 1 and block 2 are plotted separately for both sessions (session A: left graph; session $\mathrm{B}$ : right graph), indicating no significant stimulation-dependent signal changes above $9 \mathrm{~Hz}$. 
Fourier transformations and TFR calculated for both sessions and blocks separately showed an increase in signal power during the second block of session B (see Figures 2, 3), which was highest in the slow frequency bands showing a decay of $\sim 12 \mathrm{~dB}$ per decade. While not significant, we found a slight increase in broadband noise across all frequencies during anodal stimulation (second block, session B; see Figure 3B, right panel). Statistical analysis using a non-parametric cluster-based permutation test indicated significant stimulation-dependent changes of signal power in frequencies below $8 \mathrm{~Hz}$ (Figure 3C, right panel), but not in frequencies above $9 \mathrm{~Hz}$. Calculation of the stimulationdependent signal difference's effect size on each frequency band indicated a large effect on the delta band $(d=0.9893, r=0.443)$ which was weaker in the theta band $(d=0.5721, r=0.275)$ and small in the alpha $(d=0.2988, r=0.122)$ as well as beta band $(d=0.2791, r=0.115)$.

\section{BRAIN-MACHINE INTERFACE (BMI) CONTROL ACROSS SESSIONS AND CONDITIONS}

RmANOVA indicated no main effects for "session" $\left[F_{(1,9)}=\right.$ $0.30, p=0.597]$ or "block" $\left[F_{(1,9)}=1.097, p=0.322\right]$ and no interaction between the factors $\left[F_{(1,1)}=0.349, p=0.569\right]$. There also was no difference between block 1 of session $A$ and session B ( $p=0.541)$, nor a difference between block 1 and block 2 of session A $(p=0.880)$ or session B $(p=0.470)$ (Figure 4).

\section{SAFETY AND TOLERABILITY OF SIMULTANEOUS TDCS DURING EEG-BASED BRAIN-MACHINE INTERFACE (BMI) CONTROL}

Six of ten participants reported light tingling (rated at 2-3 on a VAS, mean value across all participants $1.6 \pm 0.4$ with $0=$ none and $10=$ unbearable, extreme tingling) in at least one of the sessions, and five reported light itching (mean value $1.4 \pm 0.8$; $0=$ none, $10=$ unbearable, extreme itching), mainly at the beginning of block 2. None of the participants reported any form of severe discomfort or pain. Participants were unable to distinguish sensations between session A and session B.

\section{DISCUSSION}

Our study investigated the influence of simultaneous tDCS on EEG recordings across different frequency bands and shows that online extraction of physiological signals during learned selfregulation of brain oscillations for online MI-based BMI control is feasible and safe. We found that application of tDCS is associated with a significant signal increase across slower frequency bands below $9 \mathrm{~Hz}$ (delta and theta) in direct proximity of the stimulation electrode, and delta band $(<4 \mathrm{~Hz})$ recorded at larger distance $(>\sim 8 \mathrm{~cm}$ ). However, signals oscillating above $9 \mathrm{~Hz}$ (e.g., $11 \mathrm{~Hz}$ ) were not influenced by stimulation and could be successfully used for reliable, motor imagery-based BMI control. Our results indicate that any BMI paradigm driven by modulation of brain oscillations in the alpha $(9-15 \mathrm{~Hz})$ or beta range $(15-30 \mathrm{~Hz})$ or even higher frequencies $(>30 \mathrm{~Hz})$ is possible, given that the signal/noise ratio allows proper linear or non-linear classification of the neurophysiological features used for BMI control.

An important aspect in the combination of tDCS and BMI control is the stimulation montage. Due to the conduction properties of the human head, most electric currents pass through the

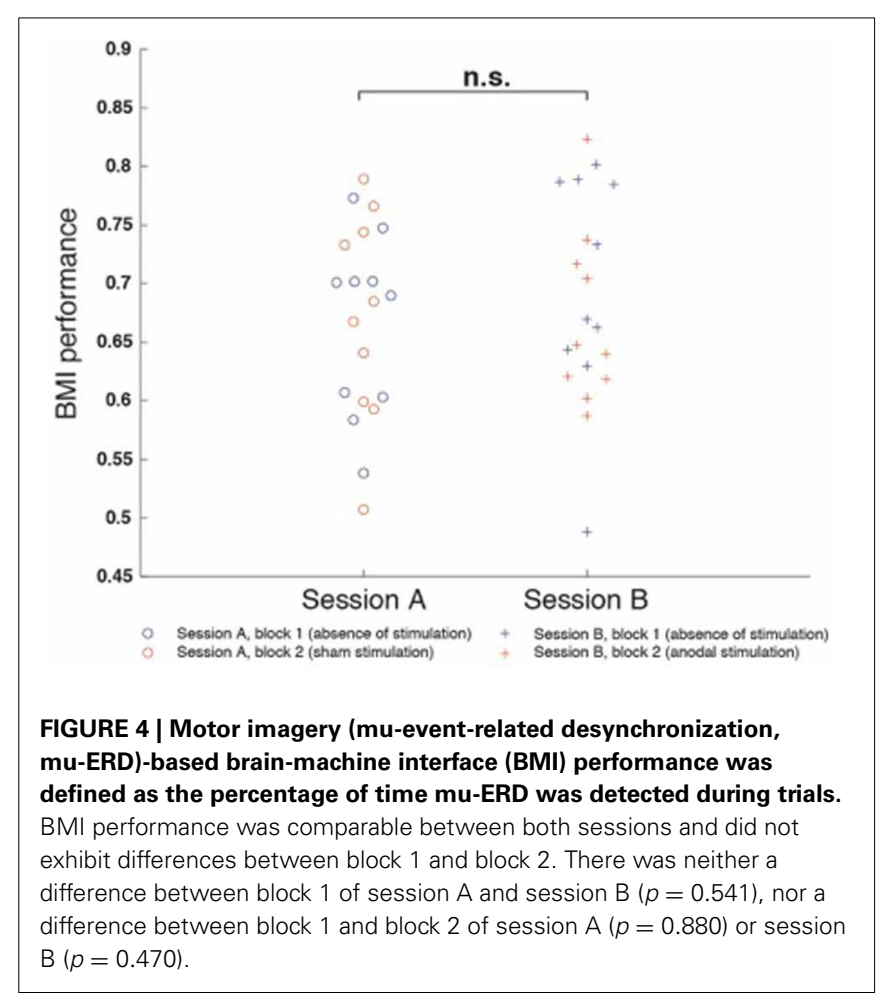

skin and cerebro-spinal fluid (CSF), while only a fraction enters the gray or white matter (Sadleir et al., 2012). The path of the electric currents in any given case depends on many individual characteristics, such as bone thickness, shape of the skull, density of bone-passing veins or volume of the outer CSF space (which is increased, for instance, in brain atrophy). Various computational models were developed to calculate intracranial current flow and to identify brain areas with the highest magnitude of cortical electric fields (Bikson et al., 2012; Sadleir et al., 2012). Depending on the montage, the area with the highest magnitude of the cortical electrical field might be in larger distance from the stimulating electrode not directly underlying the current source (Edwards et al., 2013). Thus, the technique described here which allows for the application of electric currents as close as $1 \mathrm{~cm}$ to the EEG recording channel might be used for modulating cortical activity of brain areas functionally related to BMI control.

When using a different stimulator than the one used in this investigation, corresponding characteristics of this device should be tested first before combining it with EEG-based BMI systems to rule out other stimulation-dependent contaminations of the EEG signal.

While some previous studies suggested that tDCS can have immediate effects on motor imagery-related ERD (Matsumoto et al., 2010) and BMI control (Wei et al., 2013), we did not find such immediate effects. This might be due to the fact that participants were not BMI-naive and exhibited already high and stable BMI control before admission to the study. tDCS, here applied as anodal tDCS, might not have improved BMI performance further, as a ceiling in BMI control may have been reached in these participants. Another reason could be the montage of the 
stimulation electrode placed $1 \mathrm{~cm}$ anterior of the $\mathrm{C} 4$, which might have resulted in the highest magnitude of cortical electrical fields in brain areas not related to motor imagery-based BMI control. A different placement of the stimulation electrode might have led to other results.

The immediate effects of electric currents on oscillatory activity in the human brain and their relatedness to behavior are still poorly understood (Dayan et al., 2013). As noted previously, a sensor-space based approach does not allow unambiguous differentiation between signals with a physiological origin opposed to stimulation-dependent signals deriving from the electric circuit of the stimulator. It is conceivable that the observed slow frequency signal power elevation during tDCS is in part of physiological origin and might reflect mechanisms that also underlie the previously well-described after-effects of tDCS, for instance polarityspecific modulation of cortical excitability and improvements of cognition and learning. Due to the design of the paradigm, the effect of tDCS on slow cortical potentials (SCP) could not be investigated here, but might be of interest in future studies to improve general understanding of the physiological effects of tDCS in the context of learned brain self-regulation during motor imagery-based BMI control. Implementation of algorithms with noise-canceling features, e.g., online source-reconstruction using beamformers (Soekadar et al., 2013b) might help to further investigate these mechanisms. The combination of electrical stimulation during multimodal EEG-MEG recordings in the context of brain self-regulation might help to shed light on these issues and further improve understanding of the exact neural substrates and mechanisms underlying the learning of abstract skills, like volitional modulation of brain oscillatory activity in the context of BMI control (Koralek et al., 2012).

\section{CONCLUSION}

tDCS delivered at $1 \mathrm{~mA}$ in close proximity $(1 \mathrm{~cm})$ to an EEG channel used for learned self-regulation of brain oscillatory activity above $9 \mathrm{~Hz}$ is feasible and safe. While associated with a signal power elevation across slower frequencies, brain signals above $9 \mathrm{~Hz}$ were unaffected by the stimulation allowing simultaneous application of electric currents during motor imagery-based online BMI control. Such combination might substantially improve the applicability and practicality of BMI use in patient populations, for instance, in the context of neurorehabilitation, and allow systematic investigation of the relatedness between learned brain self-regulation, brain oscillatory activity and behavior.

\section{ACKNOWLEDGMENTS}

This work was supported by the Intramural Research Program (IRP) of the National Institute of Neurological Disorders and Stroke (NINDS), USA; the Center for Neuroscience and Regenerative Medicine (CNRM), Uniformed Services University of Health Sciences, USA; the Advanced Convergence Research Center at the Daegu Gyeongbuk Institute of Science and Technology, Korea; the German Federal Ministry of Education and Research (BMBF, grant number 01GQ0831, 16SV5838K to Surjo R. Soekadar and Niels Birbaumer); the Deutsche Forschungsgemeinschaft (DFG, grant number SO932-1 to Surjo
R. Soekadar and Reinhart Koselleck Project support to Niels Birbaumer); the European Commission under the project WAY (grant number 288551 to Surjo R. Soekadar and Niels Birbaumer); the Volkswagenstiftung and the Baden-Württemberg Stiftung, Germany. We thank Sook-Lei Liew and Birgit Teufel for their assistance in preparing the manuscript.

\section{REFERENCES}

Ang, K. K., Guan, C., Chua, K. S. G., Ang, B. T., Kuah, C. W. K., Wang, C., et al. (2011). A large clinical study on the ability of stroke patients to use an EEG-based motor imagery brain-computer interface. Clin. EEG Neurosci. 42, 253-258. doi: 10.1177/155005941104200411

Bikson, M., Rahman, A., and Datta, A. (2012). Computational models of transcranial direct current stimulation. Clin. EEG Neurosci. 43, 176-183. doi: $10.1177 / 1550059412445138$

Birbaumer, N., and Cohen, L. G. (2007). Brain-computer interfaces: communication and restoration of movement in paralysis. J. Physiol. (Lond.) 579, 621-636. doi: 10.1113/jphysiol.2006.125633

Brunoni, A. R., Amadera, J., Berbel, B., Volz, M. S., Rizzerio, B. G., and Fregni, F. (2011). A systematic review on reporting and assessment of adverse effects associated with transcranial direct current stimulation. Int. J. Neuropsychopharmacol. 14, 1133-1145. doi: 10.1017/S1461145710001690

Buch, E., Weber, C., Cohen, L. G., Braun, C., Dimyan, M. A., Ard, T., et al. (2008). Think to move: a neuromagnetic brain-computer interface (BCI) system for chronic stroke. Stroke 39, 910-917. doi: 10.1161/STROKEAHA.107.505313

Cohen, J. (1988). Statistical Power Analysis for the Behavioral Sciences, 2nd Edn. Hillsdale, NJ: Lawrence Erlbaum.

Dayan, E., Censor, N., Buch, E. R., Sandrini, M., and Cohen, L. G. (2013). Noninvasive brain stimulation: from physiology to network dynamics and back. Nat. Neurosci. 16, 838-844. doi: 10.1038/nn.3422

Dockery, C.A., Hueckel-Wenig, R., Birbaumer, N., and Plewnia, C. (2009). Enhancement of planning ability by transcranial direct current stimulation. J. Neurosci. 29, 7271-7277. doi: 10.1523/JNEUROSCI.0065-09.2009

Edwards, D., Cortes, M., Datta, A., Minhas, P., Wassermann, E.M., and Bikson, M. (2013). Physiological and modeling evidence for focal transcranial electrical brain stimulation in humans: a basis for high-definition tDCS. Neuroimage 74, 266-275. doi: 10.1016/j.neuroimage.2013.01.042

Gandiga, P. C., Hummel, F. C., and Cohen, L. G. (2006). Transcranial DC stimulation (tDCS): a tool for double-blind sham-controlled clinical studies in brain stimulation. Clin. Neurophysiol. 117, 845-850. doi: 10.1016/j.clinph.2005.12.003

Koralek, A. C., Jin, X., Long, J. D. 2nd., Costa, R. M., and Carmena, J. M. (2012) Corticostriatal plasticity is necessary for learning intentional neuroprosthetic skills. Nature 483, 331-335. doi: 10.1038/nature10845

Llanos, C., Rodriguez, M., Rodriguez-Sabate, C., Morales, I., and Sabate, M. (2013). $\mathrm{Mu}-$ rhythm changes during the planning of motor and motor imagery actions. Neuropsychologia 51, 1019-1026. doi: 10.1016/j.neuropsychologia.2013.02.008

Maris, E., and Oostenveld, R. (2007). Nonparametric statistical testing of EEG-and MEG-data. J. Neurosci. Methods 164, 177-190. doi: 10.1016/j.jneumeth.2007.03.024

Matsumoto, J., Fujiwara, T., Takahashi, O., Liu, M., Kimura, A., and Ushiba, J. (2010). Modulation of mu rhythm desynchronization during motor imagery by transcranial direct current stimulation. J. Neuroeng. Rehabil. 7:27. doi: 10.1186/1743-0003-7-27

Metuki, N., Sela, T., and Lavidor, M. (2012). Enhancing cognitive control components of insight problems solving by anodal tDCS of the left dorsolateral prefrontal cortex. Brain Stimul. 5, 110-115. doi: 10.1016/j.brs.2012.03.002

Nitsche, M.A., Koschack, J., Pohlers, H., Hullemann, S., Paulus, W., and Happe, S. (2012). Effects of frontal transcranial direct current stimulation on emotional state and processing in healthy humans. Front. Psychiatry 3:58. doi: 10.3389/fpsyt.2012.00058

Nitsche, M. A., and Paulus, W. (2000). Excitability changes induced in the human motor cortex by weak transcranial direct current stimulation. J. Physiol. (Lond.) 527, 633-639. doi: 10.1111/j.1469-7793.2000.t01-1-00633.x

Nitsche, M. A., Roth, A., Kuo, M. F., and Fischer, A. K. (2007). Timingdependent modulation of associative plasticity by general network excitability in the human motor cortex. J. Neurosci. 27, 3807-3812. doi: 10.1523/JNEUROSCI.5348-06.2007 
Oldfield, R. C. (1971). The assessment and analysis of handedness: the Edinburgh inventory. Neuropsychologia 9, 97-113. doi: 10.1016/0028-3932(71)90067-4

Pellicciari, M. C., Brignani, D., and Miniussi, C. (2013). Excitability modulation of the motor system induced by transcranial direct current stimulation: a multimodal approach. Neuroimage 83, 569-580. doi: 10.1016/j.neuroimage.2013.06.076

Pfurtscheller, G., and Aranibar, A. (1979). Evaluation of event-related desynchronization (ERD) preceding and following voluntary self-paced movement. Electroencephalogr. Clin. Neurophysiol. 46, 138-146. doi: 10.1016/00134694(79)90063-4

Pfurtscheller, G., and Neuper, C. (1997). Motor imagery activates primary sensorimotor area in humans. Neurosci. Lett. 239, 65-68. doi: 10.1016/S03043940(97)00889-6

Ramos-Murguialday, A., Broetz, D., Rea, M., Läer, L., Yilmaz, O., Brasil, F. L., et al. (2013). Brain-machine interface in chronic stroke rehabilitation: a controlled study. Ann. Neurol. 74, 100-108. doi: 10.1002/ana.23879

Reis, J., Schambra, H. M., Cohen, L. G., Buch, E. R., Fritsch, B., Zarahn, E., et al. (2009). Noninvasive cortical stimulation enhances motor skill acquisition over multiple days through an effect on consolidation. Proc. Natl. Acad. Sci. 106, 1590-1595. doi: 10.1073/pnas.0805413106

Sadleir, R. J., Vannorsdall, T. D., Schretlen, D. J., and Gordon, B. (2012). Target optimization in transcranial direct current stimulation. Front. Psychiatry 3:90. doi: 10.3389/fpsyt.2012.00090

Schalk, G., McFarland, D. J., Hinterberger, T., Birbaumer, N., and Wolpaw, J. R. (2004). BCI2000: a general-purpose brain-computer interface (BCI) system. IEEE Trans. Biomed. Eng. 51, 1034-1043. doi: 10.1109/TBME.2004.827072

Schestatsky, P., Morales-Quezada, L., and Fregni, F. (2013). Simultaneous EEG monitoring during transcranial direct current stimulation. J. Vis. Exp. 76:50426. doi: $10.3791 / 50426$

Soekadar, S. R., Witkowski, M., Cossio, E. G., Birbaumer, N., Robinson, S. E., and Cohen, L. G. (2013a). In vivo assessment of human brain oscillations during application of transcranial electric currents. Nat. Commun. 4:2032. doi: 10.1038/ncomms3032

Soekadar, S. R., Witkowski, M., Mellinger, J., Ramos, A., Birbaumer, N., and Cohen, L. G. (2011). ERD-based online brain-machine interfaces (BMI) in the context of neurorehabilitation: optimizing BMI learning and performance. IEEE Trans. Neural Syst. Rehabil. Eng. 19, 542-549. doi: 10.1109/TNSRE.2011.2166809
Soekadar, S. R., Witkowski, M., Robinson, S. E., and Birbaumer, N. (2013b). Combining electric brain stimulation and source-based brain-machine interface (BMI) training in neurorehabilitation of chronic stroke. J. Neurol. Sci. 333:e542. doi: 10.1016/j.jns.2013.07.1906

Stagg, C. J., Jayaram, G., Pastor, D., Kincses, Z. T., Matthews, P. M., and JohansenBerg, H. (2011). Polarity and timing-dependent effects of transcranial direct current stimulation in explicit motor learning. Neuropsychologia 49, 800-804. doi: 10.1016/j.neuropsychologia.2011.02.009

Wang, W., Collinger, J. L., Perez, M. A., Tyler-Kabara, E. C., Cohen, L. G., Birbaumer, N., et al. (2010). Neural interface technology for rehabilitation: exploiting and promoting neuroplasticity. Phys. Med. Rehabil. Clin. N. Am. 21, 157-178. doi: 10.1016/j.pmr.2009.07.003

Wei, P., He, W., Zhou, Y., and Wang, L. (2013). Performance of motor imagery brain-computer interface based on anodal transcranial direct current stimulation modulation. IEEE Trans. Neural Syst. Rehabil. Eng. 21, 404-415. doi: 10.1109/TNSRE.2013.2249111

Zimerman, M., Heise, K.F., Hoppe, J., Cohen, L.G., Gerloff, C., and Hummel, F.C. (2012). Modulation of training by single-session transcranial direct current stimulation to the intact motor cortex enhances motor skill acquisition of the paretic hand. Stroke 43. 2185-2191. doi: 10.1161/STROKEAHA.111.645382

Conflict of Interest Statement: The authors declare that the research was conducted in the absence of any commercial or financial relationships that could be construed as a potential conflict of interest.

Received: 12 January 2014; accepted: 03 March 2014; published online: 18 March 2014. Citation: Soekadar SR, Witkowski M, Cossio EG, Birbaumer N and Cohen LG (2014) Learned EEG-based brain self-regulation of motor-related oscillations during application of transcranial electric brain stimulation: feasibility and limitations. Front. Behav. Neurosci. 8:93. doi: 10.3389/fnbeh.2014.00093

This article was submitted to the journal Frontiers in Behavioral Neuroscience.

Copyright () 2014 Soekadar, Witkowski, Cossio, Birbaumer and Cohen. This is an open-access article distributed under the terms of the Creative Commons Attribution License (CC BY). The use, distribution or reproduction in other forums is permitted, provided the original author(s) or licensor are credited and that the original publication in this journal is cited, in accordance with accepted academic practice. No use, distribution or reproduction is permitted which does not comply with these terms. 